PostGRAD. MED. J. (1965), 41, 190

\title{
A CASE OF LITHIUM INTOXICATION
}

\author{
J. L. VeRBoV, M.B., M.R.C.S. \\ Medical Registrar
J. D. Phillips, M.B., M.R.C.S. House Physician.

\author{
D. G. Fife, M.B., M.R.C.S. \\ House Physician
}

Whipps Cross Hospital, London, E.11.

LITHIUM, the lightest metal, has an atomic weight of seven and closely resembles sodium chemically. The ion is toxic and there are no therapeutic uses for lithium salts. Good (1903) quoted investigations of nearly twenty years previously in animals, where intravenous lithium salts depressed the heart's action and caused a fall in blood pressure. The dose of lithium salts necessary to stop the heart was found to be much larger than the dose of potassium salts necessary to produce the same effect.

In 1948, lithium chloride was marketed in the U.S.A. as a salt substitute (Radomski, Fuyat, Nelson and Smith, 1950). The kidney, even in severe heart failure, excretes lithium much more readily than sodium, and therefore lithium does not promote water retention and oedema formation. This fact, plus the similarity in taste between lithium and sodium chlorides led to the use of lithium as a salt substitute (Drill, 1958). However, a number of patients died after receiving lithium and it is no longer used for this purpose.

Lithium salts have been used in the treatment of mania (Lancet, 1955; Rice, 1956) and Kiloh, (1961) considered that in psychiatric practice the toxic effects of lithium salts are slight. Talbott (1950) found that symptoms of toxicity only occurred with serum lithium concentrations above $1 \mathrm{mEq}$./1. He found in a series of fourteen normal persons who ingested $1.56 \mathrm{~g}$. of lithium carbonate daily for 5-14 days that the highest lithium concentration in the serum was $1.1 \mathrm{mEq} . / 1$. In six persons gastrointestinal irritability was noted; correlation was observed between this symptom and the serum lithium. Three subjects experienced drowsiness. The mechanism whereby lithium produces its toxic effects is not clear although the principal action appears to be on the kidney.

\section{Case Report}

Miss F.F. aged 51, was admitted to Whipps Cross Hospital in coma on 7th February, 1964. Her past history revealed that in late 1956, following a broken marriage, she had been a voluntary patient in a mental hospital, where a diagnosis of recurrent attacks of schizophrenia and depressive symptoms was made. At that time, chlorpromazine and lithium carbonate therapy was started, the latter being used because of its reported value in recurrent attacks of mania. Following the introduction of lithium she had no further attacks of symptoms and in 1961 it was suggested that it should be continued in the

absence of any side-effects. Up to the time of her $\vec{\circ}$ admission to us she had been taking regularly tab. $\overrightarrow{\vec{\omega}}$ chlorpromazine hydrochloride $50 \mathrm{mg}$. three times daily and tab. lithium carbonate $250 \mathrm{mg}$. four timesdaily, although from October, 1963 she had been eating little food because of anorexia and she had?. been progressively losing weight.

On examination on admission, she was febrile (101.6), comatose, thin, with a very dry skin, and showed spasticity of her lower limbs with increased ${ }^{\triangleright}$ limb reflexes and an extensor left plantar response $\overrightarrow{0}$ There was no neck stiffness and fundi were normal. Her chest was clinically clear, pulse $96 /$ minute ando regular, BP $90 / 60 \mathrm{~mm}$.Hg. Blood was taken at once_ for serum electrolytes and other determinations, and lumbar puncture revealed a clear fluid at a normato pressure with normal cell and protein content. $A=$ Ryle's tube was introduced into her stomach and intravenous fluids were commenced at a rate of the्es litres in twenty-four hours. At first, two litres Gef. normal saline were given followed by a litre ff dextrose $(4.3 \%)$-saline $(0.18 \%)$ and then fluid wos? given according to the serum electrolyte values. Chlorpromazine and lithium carbonate were dis-o continued on admission and she was commenced on a course of intramuscular penicillin, later on in her stay receiving oral and intramuscular vitamins also. By 11th February her level of consciousness hadō improved but she was unable to co-operate; BP 140/90:3 chest X-ray at this time was clear. The Ryle's tube was taken up and intravenous fluids discontinued on the 14th February at which time she was much more alert and aware of her surroundings. Marked dys- 0 arthria was first noted on the 19th February. Towards 3 . the end of February she was slowly mobilised and showed a markedly ataxic gait. An electroencephalogram on the 9th March was essentially normal. Sheo was discharged to convalesce on the 27th April,

Investigations

Date Blood Urea $\mathrm{mg} . / 100 \mathrm{ml}$.

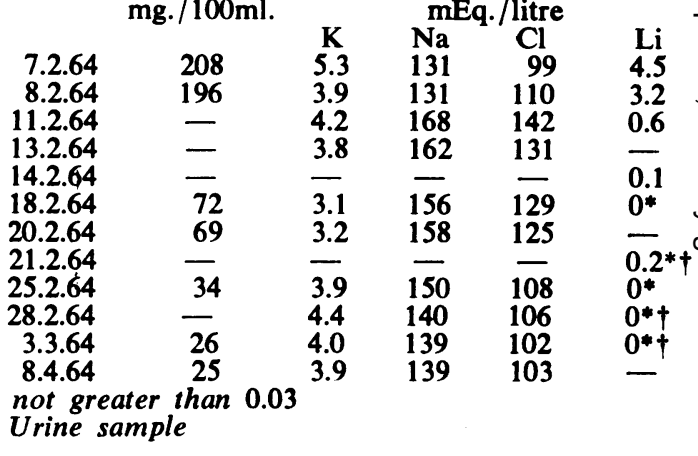


$17 \cdot 2 \cdot 64$
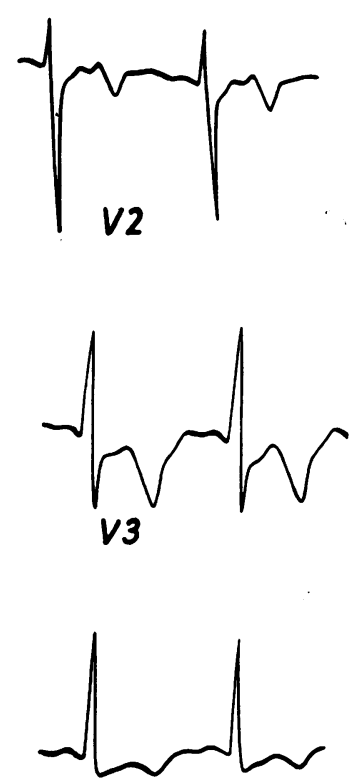

V5
$24 \cdot 2 \cdot 64$
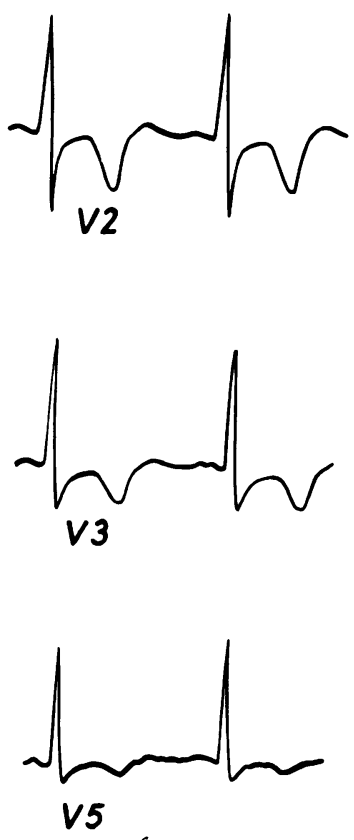

$29 \cdot 2 \cdot 64$
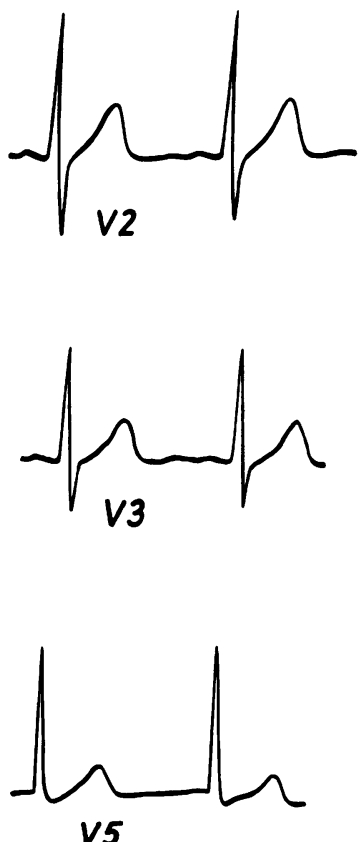

$3 \cdot 3 \cdot 64$
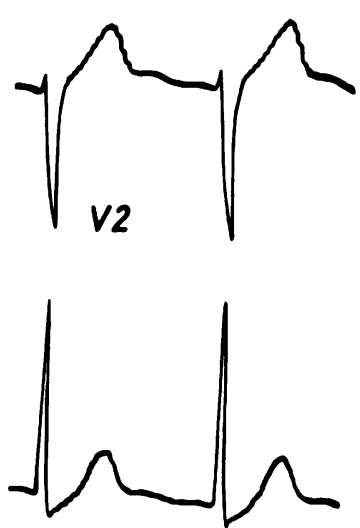

V3

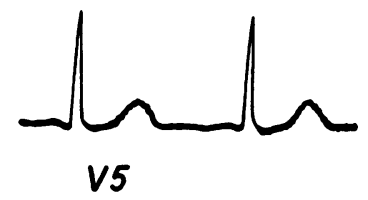

FIG. 1.-Serial Electrocardiograms.

euphoric, with some dysarthria, slightly ataxic gait, intention tremor of her right upper limb, equally brisk limb reflexes and normal plantar responses: she was eating well and felt fine. Further improvement was noted when she was seen again on the 22nd May.

\section{Discussion}

In its use as a salt substitute, Corcoran, Taylor and Page (1949) described seven cases of lithium intoxication and two of these died. They considered that sodium depletion increases the susceptibility to intoxication from oral lithium salts. Hanlon, Romaine, Gilroy and Deitrick (1949) described four cases in which lithium chloride caused toxic symptoms including weakness and drowsiness in each case. One of their patients died and four days before death when lithium was discontinued, serum lithium concentration was $4.8 \mathrm{mEq}$./1. being $3.0 \mathrm{mEq}$. $/ 1$. three days later. This patient only received lithium chloride for three days and in that time ingested approximately 13 g., complaining of generalised weakness only on the third day. Supplying sodium should relieve symptoms in patients with a high serum lithium associated with sodium depletion. The symptoms of lithium intoxication in order of their appearance include tremor, muscle twitching, apathy, drowsiness, loss of appetite, nausea, blurring of vision and confusion. Patients finally pass into a fatal coma. The symptoms parallel the lithium concentration of the blood serum. Radomski and others (1950) found in animals in the terminal stages of lithium chloride intoxication electrocardiographic changes including $T$ wave inversion with a marked increase in $T$ wave amplitude, and depression of the S-T segment: these changes were present in our patient in early tracings, reverting to normal by 29 th February (see Fig. 1). McKusick (1950) considered that the electrocardiographic effects of lithium may be secondary to its effects on the concentration of potassium in the heart muscle and in the extracellular water or in the ratio of these two.

The purpose of this paper is to draw attention to the dangers of lithium salts, particularly in the presence of an inadequate diet and to the fact that such preparations are still in use.

We should like to thank Dr. H. Wykeham Balme for his generous help, and permission to publish this case; Dr. B. T. Commins for the flame photometer lithium estimations; Dr. E. B. Grogono for details of the patient's past history; and Sister F. Morden and her staff for their care of the patient. 


\section{REFERENCES}

Corcoran, A. C., Taylor, R. D., Page, I. H. (1949): Lithium Poisoning from the Use of Salt Substitutes, J. Amer. med. Ass., 139, 685.

GARB, S. (1958): In Pharmacology in Medicine. p. 554. Edited by Drill, V. A. 2nd ed. New York: McGraw-Hill.

Good, C. A. (1903): An Experimental Study of Lithium. Amer. J. med. Sci., 125, 273.

hanlon, L. W., Romaine III. M., Gilroy, F. J., DEITRICK, J. E. (1949): Lithium Chloride as a Substitute for Sodium Chloride in the Diet, J. Amer. med. Ass., 139, 688.

KILOH, L. G. (1961): In Progress in Clinical Medicine. p. 268. 4th ed. London: J. \& A. Churchill.
LANCET (1955): Lithium salts in Manic Psychosis, $\mathbf{i}, \frac{2}{\frac{2}{2}}$ 854.

McKusicK, V. A. (1950): Effect of Lithium on the $C$ Electrocardiogram of Animals, Fed. Proc., 9, 84.

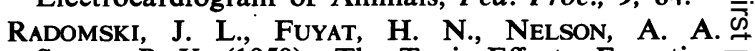
SMITH, P. K. (1950): The Toxic Effects, Excretion and Distribution of Lithium Chloride, J. Pharm. Exp. Therap., 100, 429.

RICE, D. (1956): The Use of Lithium Salts in the Treatment of Manic States, J. ment. Sci., 102, 604. 을

TALBOTT, J. H. (1950): Use of Lithium Salts as a के Substitute for Sodium Chloride, Arch. intern. Med., $\vec{\circ}$ 85, 1. 